\title{
Spatial Patterns Induced Purely by Dichotomous Disorder
}

\author{
J. Buceta and Katja Lindenberg \\ Department of Chemistry and Biochemistry, and Institute for Nonlinear Science, \\ University of California San Diego, 9500 Gilman Drive, La Jolla, CA 92093-0340, USA
}

(Dated: October 31, 2018)

\begin{abstract}
We study conditions under which spatially extended systems with coupling a la Swift-Hohenberg exhibit spatial patterns induced purely by the presence of quenched dichotomous disorder. Complementing the theoretical results based on a generalized mean-field approximation, we also present numerical simulations of particular dynamical systems that exhibit the proposed phenomenology.

PACS numbers: 05.40.-a, 47.54.+r, 05.10.Gg, 89.75.kD
\end{abstract}

\section{INTRODUCTION}

Quenched disorder and dynamical disorder play an important role in the properties of many physical systems. Some examples that illustrate this role in a variety of very different contexts and support the wide interest in the subject of disorder include the propagation of fronts in porous media 1], the conduction properties of doped semiconductors materials [2], the shift of the Curie temperature in ferromagnets [3], and the so-called Anderson localization transition [4].

At the same time, another broad field of perennial interest is pattern formation [5] because spatio-temporal structures are omnipresent in the physical world [6]. Our own recent contributions in this field involve the discovery of novel mechanisms for the appearance of spatio-temporal structures upon periodic or random global alternation of pattern-free dynamics [7].

Herein we coadunate these two topics by investigating conditions under wich the presence of quenched disorder is the mechanism that triggers pattern formation. Thus our interest lies in a particular type of purely-noise-induced phenomenon: the appearance of patterns in disordered systems which in an ordered state exhibit no pattern formation. We concentrate on systems with quenched dichotomous disorder and coupling term a la Swift-Hohenberg [8], although one can easily envision generalizations of the formalism to other kinds of disorder and/or coupling terms.

Noise-induced phenomena in spatially extended systems have been particularly active areas of investigation in the recent past [9]. Among these are phenomena involving pattern formation induced purely by fluctuations [10, 11, 12]. The word "purely" here emphasises the fact that the control parameter that determines the presence or absence of patterns is the noise intensity. Moreover, if the noise term is replaced by a non-fluctuating parameter, no spatiotemporal structures develop for any value of that parameter. Our study differs from these previous ones in that we consider quenched spatial disorder, that is, the fluctuations have no temporal dependence.

Instead of focusing on a specific system at the outset, we explore some general conditions under which these systems exhibit purely disorder induced patterns. We then illustrate our findings with a family of systems that includes the paradigmatic models of noise-induced phase transitions [13, 14, 15] and noise-induced spatial patterns 10, 13. We also identify a phenomenon in our system that has previously only been identified in systems with colored noise [14, 15, 16] or field-dependent kinetic coefficients [12], namely, a re-entrant behavior with increasing coupling strength. In other words, we find that increasing the coupling strength leads to non-monotonic behavior such that the patterns are most prominent for a finite value of the coupling and disappear altogether when coupling is too strong (or too weak). As in second and first order phase transitions in equilibrium systems, the re-entrance phenomenon can be either continuous (second order behavior) or it may present multi-stability and associated hysteresis (first order behavior).

The paper is organized as follows. We introduce the formalism in Sec. III In Sec. III and Appendix A we present a generalized mean field approximation and state the requirements for pattern formation induced purely by disorder. The possible behaviors that can be deduced from these requirements are explored in Secs. IV] and $\nabla$ Particular examples of systems that exhibit pattern formation are given in Sec. $\mathrm{VI}$ and the order parameters used to characterize the patterns are introduced and related to one another in Appendix B Numerical simulations that confirm the qualitative validity of the theoretical results are presented in Sec. VII Finally, we summarize the main results in Sec. VIII]

\section{THE MODEL}

We consider the following stochastic dynamics for a scalar field $\phi_{\boldsymbol{r}} \equiv \phi(\boldsymbol{r}, t)$ in the presence of dichotomous disorder:

$$
\dot{\phi}_{\boldsymbol{r}}=f\left(\phi_{\boldsymbol{r}}\right)+g\left(\phi_{\boldsymbol{r}}\right) \xi_{\boldsymbol{r}}+\mathcal{L} \phi_{\boldsymbol{r}}
$$


Here $\xi_{r}$ is a space dependent quenched dichotomous variable that models spatial disorder. The probability density of $\xi$ reads:

$$
\rho(\xi)=p_{+} \delta(\xi-\Delta)+p_{-} \delta(\xi+\Delta),
$$

that is, at any given site $\boldsymbol{r}$ the variable $\xi_{r}$ takes on either the value $+\Delta$ or the value $-\Delta$ with probabilities $p_{+}$and $p_{-}$respectively. The term $\mathcal{L}$ stands for the Swift-Hohenberg coupling operator

$$
\mathcal{L}=-D\left(k_{0}^{2}+\nabla^{2}\right)^{2}
$$

The effect of this coupling can be deduced by applying $\mathcal{L}$ to a plane wave $e^{i \boldsymbol{k} \cdot \boldsymbol{r}}$,

$$
\mathcal{L} e^{i \boldsymbol{k} \cdot \boldsymbol{r}}=\omega(k) e^{i \boldsymbol{k} \cdot \boldsymbol{r}},
$$

where $\omega(k)=-D\left(k_{0}^{2}-k^{2}\right)^{2}$ is the continuous dispersion relation (we use bold for vectorial quantities and italic for their magnitudes).

In order to implement a mean field theory for this system we need to distinguish from one another neighboring locations $\boldsymbol{r}$ and $\boldsymbol{r}^{\prime}$, which in turn requires that we discretize the system. Since numerical simulations also involve discretization, this procedure does not interfere with the comparisons of theoretical and numerical results. With the understanding of the action of the translation operator

$$
\exp \left(\delta x \frac{\partial}{\partial x}\right) f(x)=f(x+\delta x)
$$

it is straightforward to deduce a discrete version of the Swift-Hohenberg coupling operator $\mathcal{L}$,

$$
\mathcal{L}=-D\left[k_{0}^{2}+\left(\frac{2}{\Delta x}\right)^{2} \sum_{i=1}^{d} \sinh ^{2}\left(\frac{\Delta x}{2} \frac{\partial}{\partial x_{i}}\right)\right]^{2},
$$

where $d$ stands for the spatial dimension, $\Delta x$ for the lattice spacing, and $\frac{\partial}{\partial x_{i}}$ indicates a partial derivative with respect to component $i$ of the position vector $\boldsymbol{r}=\left(x_{1}, x_{2}, \ldots, x_{i}, \ldots, x_{d}\right)$. The continuum delta function $\delta\left(\boldsymbol{r}-\boldsymbol{r}^{\prime}\right)$ is replaced in the usual way by a ratio that contains the Kronecker delta and the lattice spacing, $\delta_{\boldsymbol{r}, \boldsymbol{r}^{\prime}} /(\Delta x)^{d}$. As in the continuous case, the discrete dispersion relation can be obtained by applying the operator (6) to a plane wave $e^{i \boldsymbol{k} \cdot \boldsymbol{r}}$, to obtain

$$
\omega(\boldsymbol{k})=-D\left[k_{0}^{2}-\left(\frac{2}{\Delta x}\right)^{2} \sum_{i=1}^{d} \sin ^{2}\left(\frac{\Delta x}{2} k_{i}\right)\right]^{2} .
$$

Here $k_{i}$ denotes component $i$ of the wave vector $\boldsymbol{k}=\left(k_{1}, k_{2}, \ldots, k_{i}, \ldots, k_{d}\right)$.

Note that $\omega(\boldsymbol{k})$ is nonpositive for any value of $k$ in both the continuous and discrete cases, and that in the discrete case it depends not only on the magnitude but also on the direction of $\boldsymbol{k}$. Of particular importance in our subsequent analysis are those modes for which $\omega(\boldsymbol{k})=0$. In the continuum these are the modes with $k=k_{0}$, which are all those that lie on a continuous hypersurface of radius $k_{0}$ around the origin. In the discretized system the magnitudes $k^{*}$ of the least stable modes are shifted from $k_{0}$ and depend on direction, as can be seen by solving Eq. (77). The longest vectors such that $\omega\left(\boldsymbol{k}^{*}\right)=0$ lie along the cartesian directions in $\boldsymbol{k}$ space, e.g. $\left(k^{*}, 0,0, \ldots, 0\right)$ and have magnitude

$$
\max k^{*}=\frac{2}{\Delta x} \arcsin \left(\frac{k_{0} \Delta x}{2}\right) .
$$

The shortest lie along a reciprocal space diagonal, e.g. $\frac{1}{\sqrt{d}}\left(k^{*}, k^{*}, k^{*}, \ldots, k^{*}\right)$, and have magnitude

$$
\min k^{*}=\frac{2 \sqrt{d}}{\Delta x} \arcsin \left(\frac{k_{0} \Delta x}{2 \sqrt{d}}\right) .
$$

In our analysis in $d=2$ we take $k_{0}=1$ and $\Delta x=1$. The difference between $\max k^{*}$ and $\min k^{*}$ is smaller than $3 \%$, the two values being $\pi / 3=1.0472$ and $\pi / 3.0737=1.0221$. Therefore it is only a mild approximation to neglect the directional dependence of the solutions of $\omega\left(\boldsymbol{k}^{*}\right)=0$ and focus on the magnitude, $\omega\left(k^{*}\right)=0$. Furthermore, in simulations one must use a finite system of $N^{d}$ sites [i.e., of volume $(N \Delta x)^{d}$ ], so that the allowed modes themselves form a discrete set, with each component separated from the next one by an interval $\delta k=2 \pi / N \Delta x$. One way to pick 
the least stable modes is to construct a ring of radius $\left\langle k^{*}\right\rangle$ (which we shall simply call $k^{*}$ from here on) of thickness $\delta k$ and to consider all the modes that lie in this ring. We can then estimate the number of modes $\mathfrak{n}\left(k^{*}\right)$ that are least stable by calculating the number of cells of volume $(2 \pi / N)^{d}$ in the ring:

$$
\mathfrak{n}\left(k^{*}\right)=\frac{d \pi^{d / 2}}{\Gamma(d / 2+1)}\left(\frac{N \Delta x k^{*}}{2 \pi}\right)^{d-1} .
$$

Although slight variations in the particular way of counting are possible, for sufficiently large $N$ the differences are small.

\section{MODULATED MEAN-FIELD THEORY}

To establish the existence of patterns of a characteristic length scale in the steady state, we seek a spatially periodic structure defined by wavevectors whose magnitude is associated with the inverse of this length scale. The appropriate wavevectors to focus on are precisely those of magnitude $k^{*}$, that is, those for which $\omega(\boldsymbol{k})=0$. A mean field theory requires that we make an ansatz about the behavior of the stationary field at sites $\boldsymbol{r}^{\prime} \neq \boldsymbol{r}$ which are coupled to the focus site $\boldsymbol{r}$ by the operator $\mathcal{L}$. We require that the ansatz capture correct limiting behaviors and also incorporate an appropriate spatial modulation. Our choice is

$$
\phi_{\boldsymbol{r}^{\prime}}=\mathcal{A}\left(k^{*}\right) \sum_{\left\{\boldsymbol{k}^{*}\right\}} \cos \left[\boldsymbol{k} \cdot\left(\boldsymbol{r}-\boldsymbol{r}^{\prime}\right)\right]+B,
$$

where the sum (or, in an infinite system, the integral) is over wavevectors of magnitude $k^{*}$. The constant $B$ is specified below. Our ansatz incorporates the assumption that all modes of magnitude $k^{*}$ contribute with equal (direction-independent) weight $\mathcal{A}\left(k^{*}\right)$. In Appendix we show that the action of the coupling operator on this ansatz is given by

$$
\mathcal{L} \phi_{\boldsymbol{r}}=D_{1}\left[\mathfrak{n}\left(k^{*}\right) \mathcal{A}\left(k^{*}\right)-\phi_{\boldsymbol{r}}\right]+B\left(D_{1}-D k_{0}^{4}\right)
$$

where

$$
D_{1}=D\left[\left(\frac{2 d}{(\Delta x)^{2}}-k_{0}^{2}\right)^{2}+\frac{2 d}{(\Delta x)^{4}}\right] \text {. }
$$

Substitution of Eq. (12) in Eq. (1) then leads to an equation that depends only on a generic site index $r$ that can simply be dropped:

$$
\dot{\phi}=f(\phi)+g(\phi) \xi+D_{1}\left[\mathfrak{n}\left(k^{*}\right) \mathcal{A}\left(k^{*}\right)-\phi\right]+B\left(D_{1}-D k_{0}^{4}\right) .
$$

In the steady state we can write the explicit equations associated with Eq. (14) as

$$
\begin{aligned}
& 0=F_{+}(\phi)+D_{1}\left[\mathfrak{n}\left(k^{*}\right) \mathcal{A}\left(k^{*}\right)-\phi\right]+B\left(D_{1}-D k_{0}^{4}\right), \\
& 0=F_{-}(\phi)+D_{1}\left[\mathfrak{n}\left(k^{*}\right) \mathcal{A}\left(k^{*}\right)-\phi\right]+B\left(D_{1}-D k_{0}^{4}\right),
\end{aligned}
$$

where we have introduced the shorthand notation

$$
F_{ \pm}(\phi)=f(\phi) \pm g(\phi) \Delta
$$

We denote the solutions of Eqs. (15) by $\phi_{ \pm}$respectively.

The amplitude $\mathcal{A}\left(k^{*}\right)$ and the constant $B$ are the mean field quantities that must be chosen self-consistently to complete the solution of the problem. To close these equations we choose

$$
B=p_{+} \widetilde{\phi}_{+}+p_{-} \widetilde{\phi}_{-}
$$

where the $\widetilde{\phi}_{ \pm}$are the steady state fields for each of the separate dynamics in completely ordered systems, that is, one with $p_{+}=1$ and one with $p_{-}=1$ (see below). Furthermore, we impose the self-consistency condition

$$
\left[\mathfrak{n}\left(k^{*}\right) \mathcal{A}\left(k^{*}\right)+B\right]=\langle\phi\rangle=\int_{-\infty}^{\infty} d \phi \phi \rho\left(\phi ; \mathcal{A}\left(k^{*}\right)\right) d \phi .
$$


Since

$$
\rho\left(\phi ; \mathcal{A}\left(k^{*}\right)\right)=p_{+} \delta\left(\phi-\phi_{+}\right)+p_{-} \delta\left(\phi-\phi_{-}\right),
$$

the self-consistency condition can be rewritten as

$$
\mathfrak{n}\left(k^{*}\right) \mathcal{A}\left(k^{*}\right)=p_{+}\left(\phi_{+}-\widetilde{\phi}_{+}\right)+p_{-}\left(\phi_{-}-\widetilde{\phi}_{-}\right),
$$

where it should be stressed that the $\phi_{ \pm}$are of course functions of $\mathcal{A}\left(k^{*}\right)$. Finally, Eqs. (15) can then be rewritten as

$$
0=F_{ \pm}\left(\phi_{ \pm}\right)+D_{1} p_{\mp}\left(\phi_{\mp}-\phi_{ \pm}\right)-D k_{0}^{4}\left(p_{+} \widetilde{\phi}_{+}+p_{-} \widetilde{\phi}_{-}\right)
$$

We are particularly interested in systems in which there are no patterns in any ordered state, that is, where patterns are purely disorder-induced (we will choose the functions $f$ and $g$ accordingly). For each value of the dichotomous disorder parameter we can write a deterministic evolution equation for the dynamics. We insist that each of these evolution equations describe a system that intrinsically has at least one steady state and hence we insist that the associated forces be confining. We also require that each of these dynamics be pattern-free, so that there must be exactly one steady state solution for each, and this steady state solution in each case must be a constant independent of $\boldsymbol{r}$. The confining condition requires that for all $\Delta$

$$
\begin{aligned}
\lim _{\phi \rightarrow-\infty} F_{ \pm}(\phi)-D k_{0}^{4} \phi & =\infty \\
\lim _{\phi \rightarrow \infty} F_{ \pm}(\phi)-D k_{0}^{4} \phi & =-\infty .
\end{aligned}
$$

Indeed, it is reasonable to require that the confinement not be due simply to the coupling. and thus to require that

$$
\lim _{\phi \rightarrow-\infty} F_{ \pm}(\phi)=\infty, \quad \lim _{\phi \rightarrow \infty} F_{ \pm}(\phi)=-\infty
$$

The steady state conditions follow directly from Eq. (1):

$$
0=F_{ \pm}(\phi)-D k_{0}^{4} \phi
$$

The solutions [already introduced in the choice of $B$ in Eq. (17)] are denoted by $\widetilde{\phi}_{+}$and $\widetilde{\phi}_{-}$respectively. Further, to avoid any insertion of patterns other than those induced by disorder, we also insist that $f(\phi)$ itself be associated with only a single steady state, i.e., that the equations $f(\phi)-D k_{0}^{4} \phi=0$ and $f(\phi)=0$ also have only a single solution. On the other hand, if we approximate the dynamics in these ordered systems by our mean field ansatz, and insist that $\mathcal{A}\left(k^{*}\right)=0$ since there are no patterns, the equations in the steady state with $B$ chosen as in Eq. (17) in each case read

$$
0=F_{ \pm}(\phi)-D_{1} \phi+\widetilde{\phi}_{ \pm}\left(D_{1}-D k_{0}^{4}\right)
$$

Clearly, the solutions of the two equations are again $\widetilde{\phi}_{+}$and $\widetilde{\phi}_{-}$respectively, and thus our choice of $B$ is consistent with the exact unpatterned solutions in the ordered steady states.

For simplicity, in most of the remainder of this work we set $p_{+}=p_{-}=1 / 2$, although we hasten to add that this condition is not necessary for the appearance of patterns. To support this statement, we show in Fig. 1 the order parameter $S\left(k^{*}\right)=\mathfrak{n}\left(k^{*}\right) \mathcal{A}^{2}\left(k^{*}\right)$ (discussed in more detail in Sec. $\nabla \mathbf{I}$ and Appendix B) vs $p_{+}$for a particular model considered later; the details of that model are not important at this point. A nonzero value of the order parameter indicates the appearance of patterns, and a higher value is indicative of more pronounced patterns. In this particular instance the strongest patterns occur when $p_{+}=1 / 2$, but the figure shows that other values of $p_{+}$also lead to pattern formation for the same model and parameter values. Furthermore, we also impose the requirement that $f(\phi)$ be an odd function and $g(\phi)$ an even function of $\phi$. This immediately leads to the symmetry

$$
F_{+}\left(\phi_{+}\right)=-F_{-}\left(-\phi_{-}\right)
$$

and, associated with the pattern-free solution $\mathcal{A}\left(k^{*}\right)=0$,

$$
\widetilde{\phi}_{+}=-\widetilde{\phi}_{-}
$$

In particular, the average homogeneous solution $B=0$. This is always one of the possible solutions of the problem. The stability of this state, and the possible appearance of other solutions with $\mathcal{A}\left(k^{*}\right) \neq 0$, are the subjects of our further analysis. 


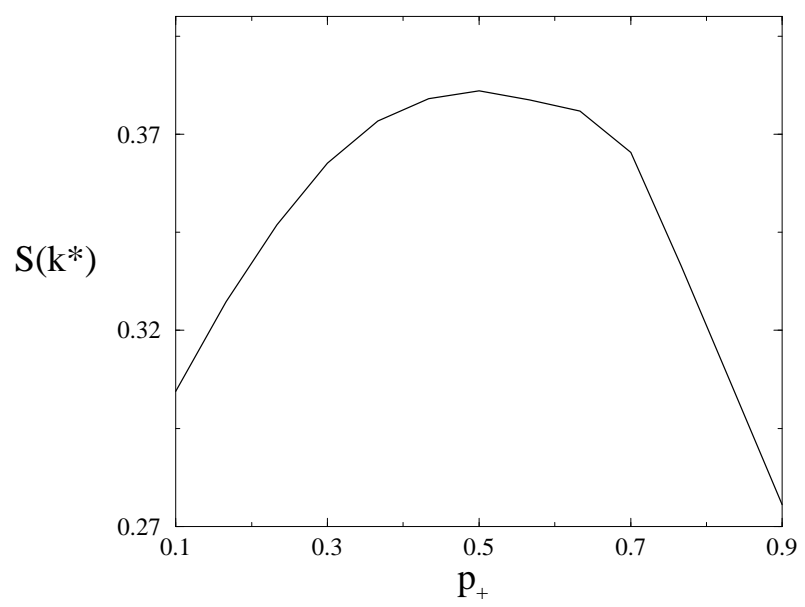

FIG. 1: Order parameter vs $p_{+}$for a model detailed in Sec. VI Pattern formation is associated with a nonzero value of the order parameter.

We collect, then, the system of equations to be analyzed with these simplifications. Furthermore, taking advantage of the symmetries established so far, the equations take on a more tractable appearance if we define new variables

$$
x \equiv \frac{D_{1}}{2} \phi_{+}, \quad y \equiv-\frac{D_{1}}{2} \phi_{-}, \quad x_{0}=\frac{D_{1}}{2} \widetilde{\phi}_{+}=-\frac{D_{1}}{2} \widetilde{\phi}_{-} .
$$

Thus the equations to solve are [cf. Eq. (15)]

$$
\begin{aligned}
& F(x)-x=y \\
& F(y)-y=x
\end{aligned}
$$

with

$$
D_{1} \mathfrak{n}\left(k^{*}\right) \mathcal{A}\left(k^{*}\right)=x-y,
$$

where we have applied the symmetry $F_{+}(x)=-F_{-}(y)$ and have dropped the subscript + since we only need to use $F_{+}$:

$$
F(x) \equiv F_{+}(x)=f(x)+g(x) \Delta .
$$

To this we add the requirement that follows from Eq. (23),

$$
\lim _{x \rightarrow \pm \infty} F^{\prime}(x)<0,
$$

where the prime denotes a derivative with respect to the argument. The solution $x=y=x_{0}$ is pattern-free. We seek solutions with $x \neq y$ to establish the appearance of patterns. Note that if a pair $(x, y)$ solves Eqs. (29), then so does the pair $(y, x)$, simply leading to a reversal in the sign of $\mathcal{A}\left(k^{*}\right)$. Since negative values of $\mathcal{A}\left(k^{*}\right)$ can be interpreted in terms of an overall spatial phase, the second pair adds no new physical information beyond the symmetry statement.

The stability of the pattern-free solution becomes an important issue in our further discussion. This can be established by a linear stability analysis around $x=y=x_{0}$. The time evolution of small perturbations about this solution is obtained by expanding the evolution equations

$$
F(x)-x-y=\dot{x}, \quad F(y)-y-x=\dot{y},
$$

at $(x, y)=\left(x_{0}+\delta x, x_{0}+\delta y\right)$ and retaining terms up to first order in the perturbations:

$$
\left(\begin{array}{c}
\delta \dot{x} \\
\delta \dot{y}
\end{array}\right)=\left(\begin{array}{cc}
F^{\prime}\left(x_{0}\right)-1 & -1 \\
-1 & F^{\prime}\left(x_{0}\right)-1
\end{array}\right)\left(\begin{array}{l}
\delta x \\
\delta y
\end{array}\right) .
$$


The $2 \times 2$ evolution matrix has eigenvalues

$$
\lambda_{+}=F^{\prime}\left(x_{0}\right), \quad \lambda_{-}=F^{\prime}\left(x_{0}\right)-2 .
$$

The largest eingenvalue (largest Lyapunov exponent) is clearly $\lambda_{+}$, and it determines the stability of the pattern-free solution:

$$
\begin{array}{ll}
F^{\prime}\left(x_{0}\right)<0 & \text { solution is stable } \\
F^{\prime}\left(x_{0}\right)>0 & \text { solution is unstable. }
\end{array}
$$

\section{PATTERN FORMATION INDUCED PURELY BY DISORDER}

We seek solutions for Eqs. (29) and, in particular, solutions with $x \neq y$. For given potential functions, one could produce three-dimensional plots of $F(x)-x-y$ and $F(y)-y-x$ vs $x$ and $y$ and observe the intersections of these surfaces. A more intuitive graphical way to organize this search is presented in the three panels of Fig. 2] The origin represents the pattern-free solution, and this is the only point on the line $y=x$ that solves the equations since $F(x)$ is not an odd function. Since Eqs. (29) are invariant under the transformation $x \longleftrightarrow y$, the line $x=y$ defines a specular plane, that is, $F(y)-y$ is a specular image of $F(x)-x$ with respect to that symmetry plane. The asymptotic behavior (32) tells us that for sufficiently large $|x|$ in the upper left quadrant, $F(x)-x$ lies above the line $x=-y$ and has a slope $<-1$. This is schematically indicated by the thick solid line in the upper left quadrant of each panel in the figure. Similarly, in the lower right quadrant the thick solid line recognizes that $F(x)-x$ must lie below the line $x=-y$ with a slope $<-1$. The specular symmetry around the line $x=y$ then leads us to the asymptotic thick dashed lines representing the behavior of $F(y)-y$.

In panel (a) we illustrate a case for which the pattern-free solution is stable, that is, $F^{\prime}\left(x_{0}\right)<0$. This is indicated by the thick solid line going through the origin. Again, the specular symmetry leads us to the thick dashed line to indicate the approriate slope for $F(y)-y$. Now it is clear that the thick solid lines can be connected, and the thick dashed lines can be connected, in such a way that the two lines do not cross anywhere else but at the origin. Thus, when the state $\left(x_{0}, x_{0}\right)$ is stable, there may not appear any other stationary states and the system may simply be pattern free. This is the case we have sketched in panel (a).

On the other hand, it is possible to connect the solid and dashed curves respectively in such a way that there are two additional crossings of the curves (actually four crossings, but only two provide independent information). These represent two additional steady state solutions, each leading to pattern formation since $x \neq y$. However, only one of the two is stable. This is illustrated in panel (b). This represents the case of coexisting stable states, one pattern-free and the other patterned, separated by an unstable state. Such coexisting states are characteristic of first order phase transitions. One can carry this further and envision further crossings, always an even number of additional crossings, representing stable patterned states that coexist with one another and with the pattern-free state, separated by unstable states.

In panel (c) we illustrate a case for which the pattern-free solution is unstable, that is, $F^{\prime}\left(x_{0}\right)<0$. Again, this is indicated by the thick solid line going through the origin together with its dashed specular partner. Now it is clear that a connection of the lines necessarily leads to at least one crossing with $x \neq y$. In other words, when the pattern-free state becomes unstable, at least one patterned state is necessarily stable. Therefore, a sufficient condition for the occurrence of patterns is that the pattern-free state become unstable.

In summary, we have found the following general behavior:

- When the pattern-free solution is stable, there may or may not occur one (or more) stable solution(s) that leads to pattern formation, the patterned and unpatterned stable states being separated from one another by unstable solutions. The appearance of such an additional stable solution is indicative of a first order phase transition, with the usual coexistence and hysteresis characteristics.

- On the other hand, when the pattern-free solution is unstable, a patterned stable state necessarily appears. The destabilization of the pattern-free state might simply mark the end of the coexistence region of a first-order transition described above, or it might mark the occurrence of a second order transition. These alternatives are discussed in further detail below.

We note here that although we have not said so explicitly, it is implicit in this entire analysis that patterned states can only exist if the potential functions are nonlinear. Linear forms can not satisfy the conditions that lead to the emergence of patterns. 


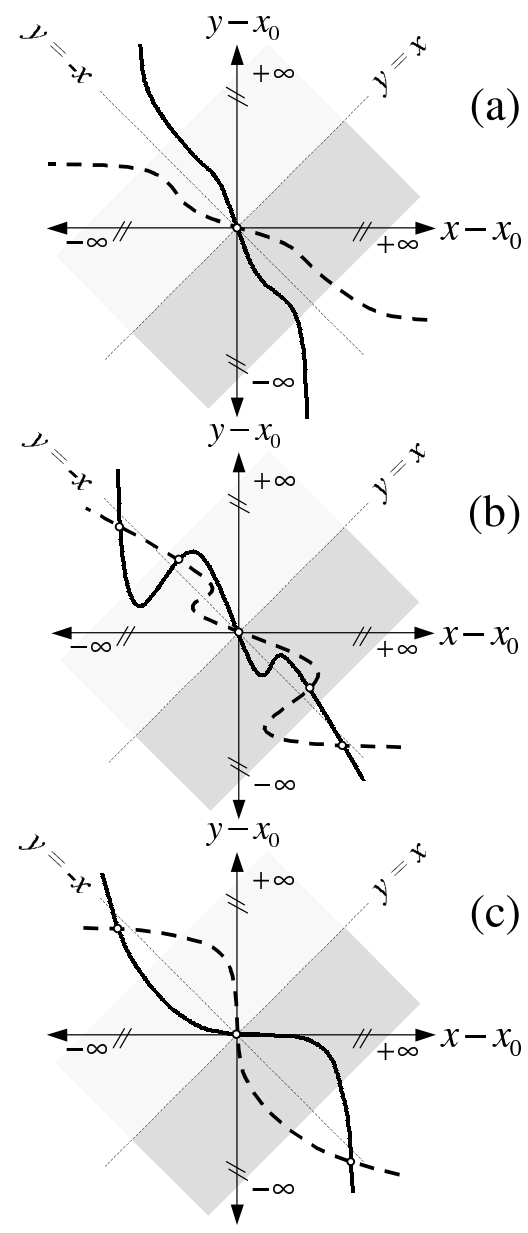

FIG. 2: Schematic of possible solutions of the mean field equations. The origin represents the pattern-free solution. Other intersections of the thick solid curve (which represents $F(x)-x$ ) and the thick dashed curve (which represents $F(y)-y$ ) are solutions of Eq. (29) that lead to patterns. Panel (a) represents a case in which the pattern-free solution is stable and there are no other solutions, that is, there is no pattern formation. Panel (b) describes the coexistence of the stable pattern-free state with a patterned stable state. In panel (c) the pattern-free state is unstable and the only stable state is a patterned state.

\section{MEAN FIELD SOLUTION - GENERAL FEATURES}

Another way to exhibit the variety of possible transitions discussed above is to focus on the explicit solution of the mean field equations and consider the resulting values of $\mathcal{A}\left(k^{*}\right)$. We start with the first Eq. (29) and subtract $x$ from both sides, $F(x)-2 x=y-x$. Similarly, we subtract $y$ from both sides of the second equation, to write $F(y)-2 y=x-y$. Using Eq. (30) then implies that we can write

$$
\begin{aligned}
D_{1} \mathfrak{n}\left(k^{*}\right) \mathcal{A}\left(k^{*}\right) & =-F(x)+2 x \equiv H_{-}(x) \\
& =F(y)-2 y \equiv H_{+}(y) .
\end{aligned}
$$

We can invert these relations,

$$
x=H_{-}^{-1}\left(\mathcal{A}\left(k^{*}\right)\right), \quad y=H_{+}^{-1}\left(\mathcal{A}\left(k^{*}\right)\right) .
$$

The self consistency condition (30) for the mean field solution can then be written as

$$
D_{1} \mathfrak{n}\left(k^{*}\right) \mathcal{A}\left(k^{*}\right)=\left[H_{-}^{-1}\left(\mathcal{A}\left(k^{*}\right)\right)-H_{+}^{-1}\left(\mathcal{A}\left(k^{*}\right)\right)\right] \equiv D_{1} \mathfrak{n}\left(k^{*}\right) G\left(\mathcal{A}\left(k^{*}\right)\right) .
$$

Since $\mathcal{A}\left(k^{*}\right)=0$ is always a solution (albeit not always stable), we know that $G(0)=0$. Furthermore, the symmetry of the problem implies that if $\mathcal{A}\left(k^{*}\right)$ is a solution, then so is $-\mathcal{A}\left(k^{*}\right)$. Therefore $G\left(\mathcal{A}\left(k^{*}\right)\right)$ is an odd function. An 


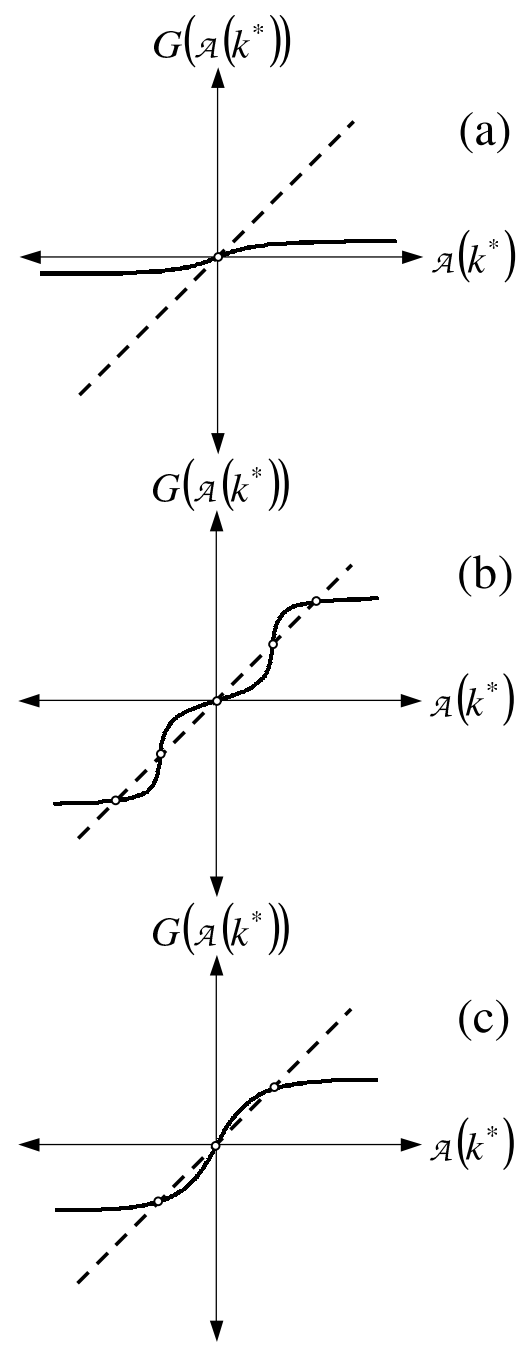

FIG. 3: Schematic of $G(\mathcal{A})$ vs $\mathcal{A}$. Intersections of the thick solid curve and the thick dashed line represent solutions of the mean field equations. Each panel represents the same situation as the corresponding panel in Fig. 22 Panel (a) depicts the case of a stable pattern-free solution and no other. Panel (b) shows the case of coexistence of the stable pattern-free state and a patterned stable state (separated by an unstable solution). In panel (c) the pattern-free state is unstable and only a patterned state is stable.

expansion about zero thus has only odd powers,

$$
G\left(\mathcal{A}\left(k^{*}\right)\right)=a \mathcal{A}\left(k^{*}\right)+b \mathcal{A}^{3}\left(k^{*}\right)+\mathcal{O}\left(\mathcal{A}^{5}\left(k^{*}\right)\right) .
$$

Our analysis proceeds on the basis of the first term of this expansion as well as the asymptotic behavior of the function $G$. The graphical representation of this analysis is shown in the three panels of Fig. 3 each associated with the corresponding panels in Fig. 2

Let us first deduce the asymptotic behavior of $G$ by considering the slope

$$
\frac{\partial H_{-}^{-1}(\mathcal{A})}{\partial \mathcal{A}}=\frac{1}{\frac{d}{d x} H_{-}(x)}=\frac{1}{\frac{d}{d x}[-F(x)+2 x]}=\frac{1}{\left[2-F^{\prime}(x)\right]},
$$

where we have applied the general relation

$$
\frac{d}{d z} h^{-1}(z)=\frac{1}{h^{\prime}\left[h^{-1}(z)\right]}
$$


and the prime, as usual, denotes a derivative with respect to the argument. In particular, we thus find that

$$
\lim _{\mathcal{A} \rightarrow \pm \infty} \frac{\partial H_{-}^{-1}(\mathcal{A})}{\partial \mathcal{A}}=\lim _{x \rightarrow \pm \infty} \frac{1}{\left[2-F^{\prime}(x)\right]}<\frac{1}{2}
$$

where we have used Eq. (32). The slope $-\partial H_{+}^{-1}(\mathcal{A}) / \partial \mathcal{A}$ leads to exactly the same asymptotic result, so that it follows from Eq. (39) that

$$
\lim _{\mathcal{A} \rightarrow \pm \infty} \frac{\partial G\left(\mathcal{A}\left(k^{*}\right)\right)}{\partial \mathcal{A}\left(k^{*}\right)}<1 .
$$

This clearly implies that asymptotically the function $G(\mathcal{A})$ in the positive half-plane must lie below the diagonal line, as we have drawn in the three panels in Fig. 3 (in the negative half plane it must lie above, again as shown). We keep in mind that patterned solutions are associated with intersections of the function $G(\mathcal{A})$ and the diagonal, away from the origin.

Next we look at the behavior of $G$ near the origin, and consider the coefficient $a$ in the Taylor expansion (40). The calculation of this coefficient involves precisely the steps followed above, but with the functions evaluated at $x_{0}$ instead of asymptotically. We readily obtain

$$
\left.a \equiv \frac{\partial G\left(\mathcal{A}\left(k^{*}\right)\right.}{\partial \mathcal{A}\left(k^{*}\right)}\right|_{\mathcal{A}\left(k^{*}\right)=0}=\frac{2}{2-F^{\prime}\left(x_{0}\right)}
$$

Now suppose that $F^{\prime}\left(x_{0}\right)<0$, a condition that according to Eq. (36) means that the pattern-free solution is stable. The slope of $G\left(\mathcal{A}\left(k^{*}\right)\right)$ near the origin is below the diagonal, $a<1$. It is then possible that other than the crossing at the origin there is no other crossing, that is, only the stable pattern-free solution exists. This is illustrated in panel (a) of Fig. 3, and corresponds to the situation in panel (a) of Fig. 2

Another possibility is that there are an even number of additional crossings, as illustrated in panel (b). One of the two additional solutions shown in the panel would be stable, the other unstable, and the stable patterned solution would coexist with the pattern-free solution under the circumstances shown in the panel. Again, this corresponds to the situation in panel (b) of Fig. 2 Below we establish further conditions on the forces that might lead to this behavior.

Next suppose that $F^{\prime}\left(x_{0}\right)>0$, the condition that according to Eq. (36) is associated with an unstable pattern-free solution. The slope of $G\left(\mathcal{A}\left(k^{*}\right)\right)$ near the origin is now above the diagonal in the positive half-plane, $a>1$, and another crossing than the one at the origin certainly occurs, thus insuring a stable patterned solution. This is shown in panel (c) of Fig. [3 and corresponds to panel (c) in Fig. 2]

Although neither a necessary nor a sufficient condition, it is apparent that the additional crossings in panel (b) of Fig. 3 might be accompanied by a positive curvature of $G(\mathcal{A})$ near the origin, as shown in the panel, that is, additional crossings might occur if the coefficient $b$ in Eq. (40) is positive. Likewise, case (c) is likely to be associated with a negative curvature near the origin, $b<0$.

It is useful to take cognizance of the possible sequences of behavior as one varies model parameters. For instance, a sequence $(\mathrm{a}) \rightarrow(\mathrm{b}) \rightarrow$ (c) would signal a first order phase transition from an unpatterned state through a coexistance regime of unpatterned and patterned states to a regime where only the patterned state is stable. On the other hand, a sequence (a) $\rightarrow$ (c) would represent a second order phase transition from an unpatterned to a patterned state. Transitions might be re-entrant, so that a returns $(\mathrm{c}) \rightarrow(\mathrm{b}) \rightarrow$ (a) (first order) or (c) $\rightarrow$ (a) (second order) are possible. In the next section we explore these results in the context of particular examples.

\section{MEAN FIELD SOLUTION - PARTICULAR EXAMPLES AND PHASE DIAGRAMS}

Consider the particular family of force functions

$$
f(\phi)=-\phi\left(1+\phi^{2}\right)^{m}, \quad g(\phi)=\left(1+\phi^{2}\right)^{m},
$$

with $m \geq 1$. We will concentrate in particular on the cases $m=1$ and $m=2$.

It is straightfoward to check that with this family of force functions, for any value of $\Delta$ and coupling $D$, Eqs. (24) have only a single real solution, that is, each of the ordered systems is pattern-free in the steady state. Thus, any pattern observed in the disordered system is purely a consequence of the disorder.

We start with $m=1$, a choice that has been made in a number of studies of purely noise induced transitions 13, 14, 15]. This is a particularly useful example because it can be solved analytically in mean field. Furthermore, We solve 


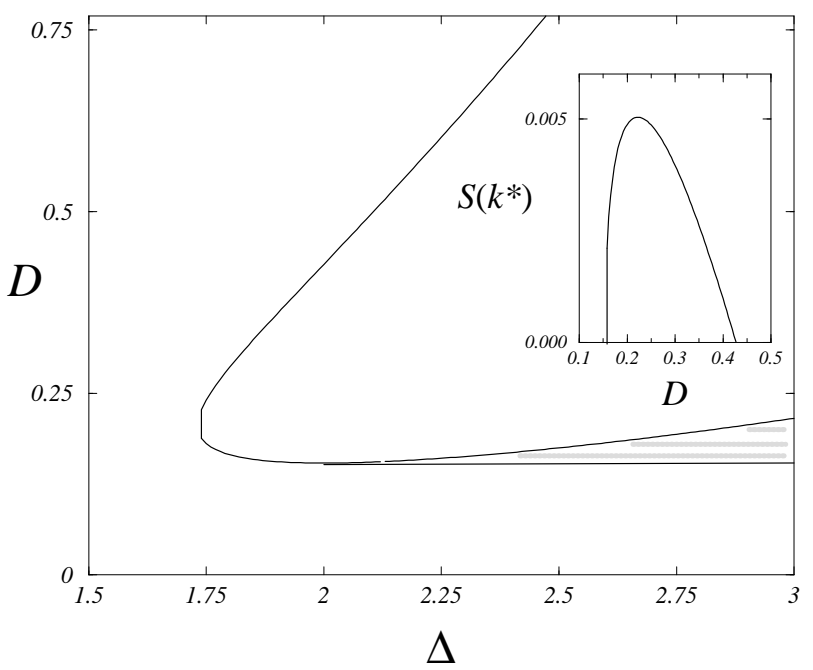

FIG. 4: Phase diagram for the case $m=1$. The shaded part of the diagram is a coexistence region and the unshaded interior denotes the occurrence of a single patterned phase. See text for a detailed description. The inset shows the behavior of the order parameter defined in the text as a function of $D$ along the line $\Delta=2$.

the coupled equations (29) fully and calculate the resulting amplitude $\mathcal{A}$ using Eq. (30), check the solutions for their stability properties, and thus construct a phase diagram as a function of the parameters $\Delta$ and $D$. This procedure

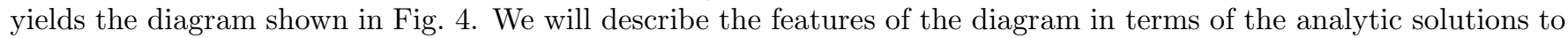
the problem as well as the general diagnostic measures described in the previous sections.

Equations (29) are cubic and yield altogether five solutions. One is the pattern-free solution $x_{0}=y_{0}$ for which $\mathcal{A}\left(k^{*}\right)=0$. Of the remaining four solutions, only two are distinct (the other two are their negatives) and they yield:

$$
\mathfrak{n}\left(k^{*}\right) \mathcal{A}_{ \pm}\left(k^{*}\right)= \pm \frac{1}{2}\left(\Delta^{2} \pm \Delta \sqrt{2\left(D_{1}-2\right)}-\frac{3}{2} D_{1}-1\right)^{1 / 2}
$$

Note that in our equations we continue to use $D_{1}$ for aesthetic reasons, whereas our illustrations involve $D$. In two dimensions with the parameters specified earlier, the two are related by $D_{1}=13 D$. Several features of these solutions are noteworthy and describe the results in Fig. 4

1. Both solutions are complex if $D_{1}<2$. Therefore, the only stationary state when $D_{1}<2$ is pattern-free (panels (a) in Figs. 2 and 3 ).

2. When $D_{1} \geq 2$, both solutions are real in the parameter range

$$
\Delta>\frac{1}{2}\left(\sqrt{2\left(D_{1}-2\right)}+2 \sqrt{2 D_{1}}\right) .
$$

One of these solutions $\left(\mathcal{A}_{+}\right)$is stable, the other unstable, and this regime marks the shaded wedge in the figure, where the stable patterned solution coexists with the pattern-free solution. Entry into this region marks a first order phase transition (panels (b) in Figs. 2] and 3). The two solutions merge (and the wedge closes) at the point $\Delta=2, D_{1}=2$.

3. When $D_{1} \geq 2$ and

$$
\frac{1}{2}\left(-\sqrt{2\left(D_{1}-2\right)}+2 \sqrt{2 D_{1}}\right)<\Delta<\frac{1}{2}\left(\sqrt{2\left(D_{1}-2\right)}+2 \sqrt{2 D_{1}}\right),
$$

only the solution $\mathcal{A}_{+}$is real. This solution is stable, and delimits the unshaded region within which there is only a single steady state, which is patterned. The transition into this regime is part of the first order phase transition if the crossing is from the shaded region, or a second order transition if from the pattern-free region (panels (c) in Figs. 2 and 3). The boundary of this regime is thus precisely the curve defined by the bounds given in Eq. (49). Alternatively, we can invert this equation and express these same bounds as

$$
D_{1}=\frac{2}{9}\left(5 \Delta^{2}-3 \pm 4 \Delta \sqrt{\Delta^{2}-3}\right) .
$$




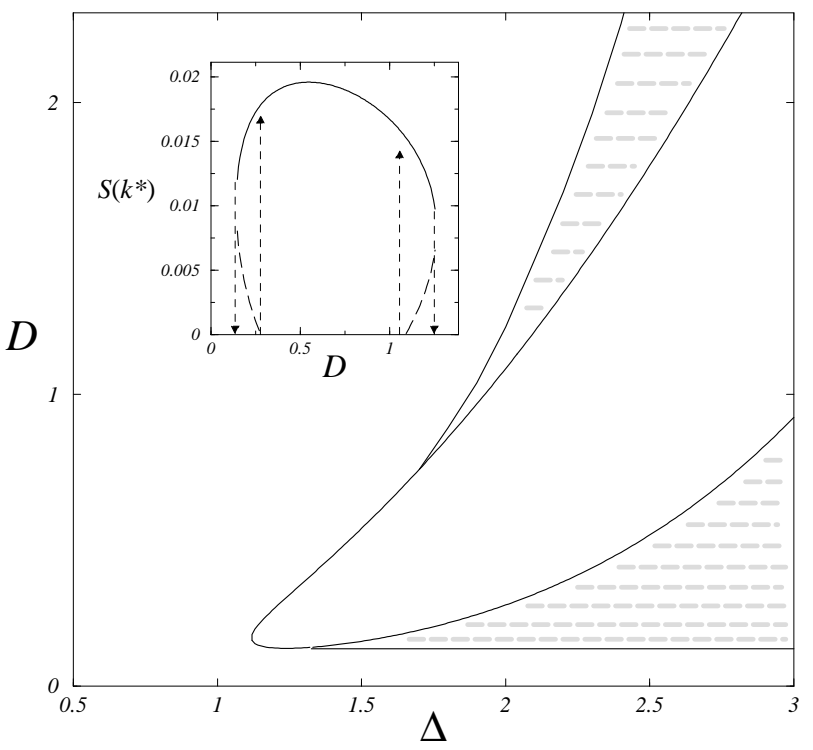

FIG. 5: Phase diagram for the case $m=2$. As in the case $m=1$, the shaded portions represent coexistence regions and the unshaded interior indicates a single patterned phase. The inset graph shows the order parameter as a function of $D$ for $\Delta=2$. Note the double-hysteresis behavior as a function of the coupling.

Note that the two curves meet at the point $\Delta=\sqrt{3}, D_{1}=8 / 3$, which is the leftmost point of the contour.

4. According to this description, the boundary of the unshaded region is precisely the curve along which $F^{\prime}\left(x_{0}\right)=0$, thus bounding the regime within which the pattern-free stationary state becomes unstable. This is indeed the case, as we can establish without solving explicitly for $x_{0}$ as a function of $D_{1}$ and $\Delta$ (the result is rather cumbersome). In terms of our original variable, for our potential we have the explicit expression $F^{\prime}(\phi)=-3 \phi^{2}+2 \Delta \phi+1$. Setting this to zero yields $\widetilde{\phi}_{+}=\left(\Delta \pm \sqrt{\Delta^{2}-1}\right) / 3$. Requiring that these values indeed be ones that solve Eqs. (29) with $x=y=x_{0}$ or, in our original notation, $F\left(\widetilde{\phi}_{+}\right)=D_{1} \widetilde{\phi}_{+}$, again gives exactly Eq. (50).

We call special attention to the striking re-entrance behavior of pattern formation as a function of the coupling $D$ : sufficiently strong coupling destroys any patterns. Note that this implies that for a given value of the disorder parameter $\Delta$ there is an optimal coupling for which the patterned structure is most pronounced. A more nuanced discussion of this behavior requires quantification in terms of order parameters. In Appendix B we introduce in Eqs. (B6) and (B7) the total power spectrum $S(k)$ and the flux of convective heat $J$. They are related to one another in Eq. (B8), and they both contain useful information. In general, when there is no pattern at all, $S(k)$ is independent of $k$ and of $\mathcal{O}(J / N)$, i.e., $S(k) / J=\mathcal{O}(1 / N)$ for all $k$. On the other hand, when there is a pattern of wavevector magnitude $k^{*}$, then $S\left(k^{*}\right)$ is of $\mathcal{O}(J)$, i.e., $S\left(k^{*}\right) / J=\mathcal{O}(1)$. A larger value of $S\left(k^{*}\right)$ indicates a stronger pattern. On the other hand, a larger ratio $S\left(k^{*}\right) / J$ indicates a more coherent pattern. It is possible (as we shall see below) for a pattern to become more (or less) coherent even as it becomes less (or more) pronounced.

In our mean field theory, however, we do not have access to all of this information because we do not deal with all modes but only with those that dominate the pattern when one is present. We are therefore restricted to choose as an order parameter the value of the power spectrum at $k^{*}$ :

$$
S\left(k^{*}\right)=\mathfrak{n}\left(k^{*}\right) \mathcal{A}^{2}\left(k^{*}\right) .
$$

Within our theory $S\left(k^{*}\right)$ and $J$ are the same. When there is no pattern they are both zero and their ratio is undefined. When there is a pattern, $S\left(k^{*}\right) / J=1$. Our theory can therefore predict whether a pattern will become stronger as parameters are modified, but not whether it will become more or less coherent.

The inset in Fig. [4 shows the power spectrum for the $m=1$ model as a function of coupling at $\Delta=2$ obtained from our theory. The values are just at the edge of the second order phase transition region and, accordingly, the order parameter rises continuously (albeit steeply) from zero upon entry into the patterned region. It also vanishes continuously as we exit the patterned region, again indicating a second order transition from the patterned state back to a pattern-free state (re-entrance) with increasing coupling. The most pronounced pattern is associated with the maximum in this curve, which can be found from Eq. (47) to occur at $D_{1}=2.94$.

We have also carried out the same set of calculations for the potential functions (46) with $m=2$. While some 
phase boundary information can be obtained analytically, full analytic solution is no longer possible because the equations are now quintic. A numerical solution is straightforward and leads to the phase diagram shown in Fig. 5 The inset shows the order parameter for $\Delta=2$, where the appearance and disappearance of patterns with increasing coupling are both first-order transitions. As a result, the order parameter curve jumps discontinuously at both ends and exhibits, at both ends, the usual hysteresis behavior associated with first-order transitions.

\section{NUMERICAL SIMULATIONS}

In order to check the predictions of the modified mean field theory we perform numerical simulations for the family of force functions given in Eq. (46) with $m=1$ and $m=2$. We implement an Euler numerical scheme on a two dimensional square lattice with periodic boundary conditions. The values of the parameters used in the simulations are $\Delta x=1, k_{0}=1, L=N \Delta x=64$, and $\Delta t=10^{-3}$. The aspect ratio, which measures the number of wavelengths of the expected patterns that fit into the system, is $L /\left(2 \pi / k^{*}\right) \sim 10$. Below we typically present averages over ten disorder configurations.

Pattern formation is characterized by the total power spectrum, $S\left(k^{*}\right)$ [cf. Eq. (B6)], where the sum runs over wavevectors $\mathbf{k}$ whose magnitude $k$ lies in the interval $\left[k^{*}-2 \pi / L, k^{*}+2 \pi / L\right]$. In addition to this parameter and the flux of convective heat $J$, another useful quantity for characterizing the system is the angular average of $\chi(\mathbf{k}) \equiv \widetilde{\phi}_{\mathbf{k}} \widetilde{\phi}_{-\mathbf{k}}$.

The first panel in Fig. [6] shows the order parameter $S\left(k^{*}\right)$ as a function of the disorder intensity parameter $\Delta$ for the family $m=1$ and for coupling coefficient $D=5$. In the absence of disorder there is no pattern, $S\left(k^{*}\right) \approx 0$, but a pattern clearly develops as $\Delta$ increases and is therefore entirely due to the disorder. The inset graphs show $\chi(\mathbf{k})$ by means of a wavevector density plot for $\Delta=3.5$ and for $\Delta=5$. Clearly, a ring of unstable modes develops around $k^{*}$, and the ring becomes more prominent with increasing disorder (as measured by the value of $\Delta$ ). While we recognize that our modified mean field theory does not predict the transition values quantitatively (for $D=5$ we predict a patterned state to first appear when $\Delta=11.5$ while the simulations already show pattern formation for much smaller values of $\Delta$ ), the qualitative behavior is as predicted. It should be noted that while the intensity of $\chi(\mathbf{k})$ at the most unstable modes increases as indicated by the gray scale, the width of the ring around $k^{*}$ also increases with increasing $\Delta$. While a higher intensity indicates a more pronounced pattern, the width is a measure of the coherence of the patterns, increasing width indicating greater decoherence. As mentioned earlier, the ratio $S\left(k^{*}\right) / J$ can be used to characterize the coherence of the patterns. This nonmonotonic ratio is shown in the second panel in Fig. 6. As also noted earlier, this ratio can not be obtained from our mean field theory.

The re-entrant behavior as a function of the coupling predicted by the modified mean field theory is shown in the first panel of Fig. 7 for $m=1$ and $\Delta=2.5$. The inset panels depict density plots of $\chi(\mathbf{k})$ for $D=0, D=2$, and $D=12$. Again, the agreement with the theory is only qualitative but nevertheless dramatic because re-entrance with increasing coupling strength is a rare phenomenon. Notice the destabilization of a ring of modes around $k^{*}$ with increasing coupling, and its subsequent intensity fade-out as $D$ increases further. It should be noted that not only does the intensity of $\chi(\mathbf{k})$ at the most unstable modes decrease as indicated by the gray scale, but the width of the ring also shrinks around $k^{*}$ as the coupling grows. The patterns thus become less pronounced but more coherent with increasing coupling. A representation of $S\left(k^{*}\right) / J$, shown in the second panel of Fig. 7 reveals that although the system presents a re-entrant behavior with the coupling, the coherence actually increases monotonically as a function of $D$.

The actual stationary spatial patterned configuration induced by the disorder is shown in the lower panel of Fig. 8 for $m=1, \Delta=2.5$ and $D=5$, i.e., the rightmost point in the first panel of Fig. 7 The upper panel in Fig. 8 shows the particular configuration of quenched disorder in the simulation that leads to the stationary field shown in the lower panel.

We have thus confirmed the disordered-induced pattern formation phenomenon but have not yet ascertained our other prediction, namely, the occurrence of hysteresis in some cases. We predict hysteresis to occur in the shaded regions of the phase diagrams in Figs. 4 and 5 Although we do not necessarily expect quantitative agreement with the particular values of parameters that lead to hysteresis, the question is whether hysteresis is observed at all.

Hysteresis implies a memory of the initial conditions. To detect hysteresis we carry out two simulations. In one, starting from an unpatterned initial condition $\phi_{\mathbf{r}}\left(t_{0}\right)=0$ for all $\mathbf{r}$, we calculate the total power spectrum in the steady state as a function of increasing coupling. Then we decrease the coupling along the same phase space path, but now our initial condition for each value of the coupling is the stationary state obtained in the simulation with the previous value of $D$. A difference in the spectrum obtained for these two different conditions reflects hysteresis and the attendant coexistence of different stationary states (one patterned and one unpatterned, as discussed earlier). In Fig. 9] we observe precisely this behavior for the family of functions $m=2$ when increasing (black circles) and decreasing (white circles) the coupling for a fixed value of the intensity of the disorder, $\Delta=3$. Hysteresis is observed between $D=0$ and $D \sim 7$. The qualitative behavior is thus exactly as predicted by the mean field model. 

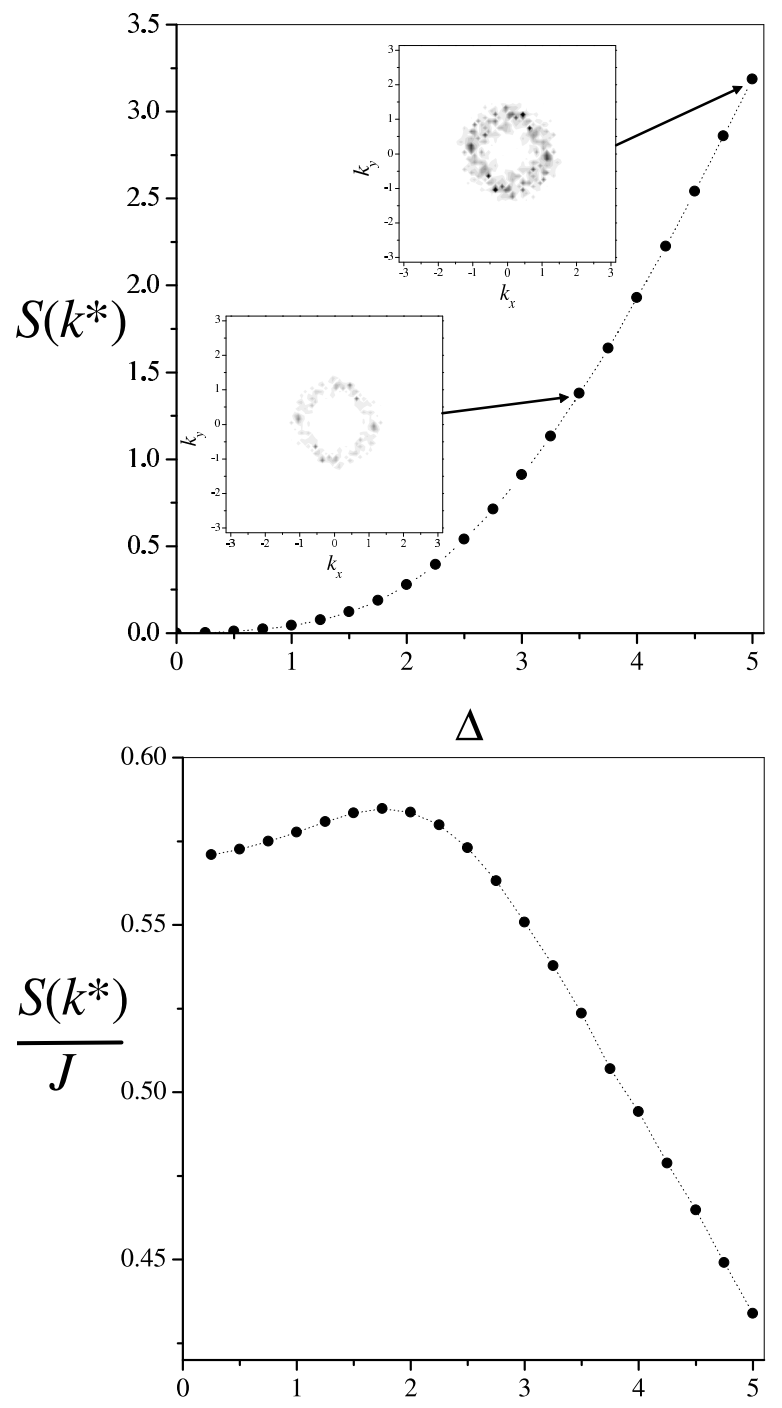

$\Delta$

FIG. 6: First panel: $S\left(k^{*}\right)$ vs $\Delta$ for $m=1$ and $D=5$. The insets are density plots of $\chi(\mathbf{k})$ for $\Delta=3.5$ and for $\Delta=5$. Note the destabilization of the $k^{*}$-modes as $\Delta$ grows. The gray scale used in the density plot is the same for both insets. Second panel: $S\left(k^{*}\right) / J$ vs $\Delta$. Note the nonmonotonic behavior, indicating maximal coherence at around $\Delta=2$.

The coexistence of patterned and unpatterned states is discernible in the density plot insets of $\chi(\mathbf{k})$ in the figure in that they are no longer cleanly annular but now include contributions from wavevectors other than those of magnitude near $k^{*}$. The difference between the two insets (indicating different relative contributions of patterned and unpatterned states) is visible but, an even clearer rendition of the difference is seen in the angular average $\langle\chi(\mathbf{k})\rangle_{\theta}$ shown in the other two inset panels. Note, for instance, that the contribution of modes near $k=0$ are relevant when increasing the coupling but very small when decreasing $D$.

\section{SUMMARY}

Using a modified mean field theory, we have explored general conditions under which spatially extended systems with coupling a la Swift-Hohenberg exhibit pattern formation purely induced by the presence of quenched dichotomous disorder. We have illustrated the phenomenology with a family of force functions that includes the paradigmatic models of noise-induced phase transitions and noise-induced spatial patterns, among them one that can fully be 

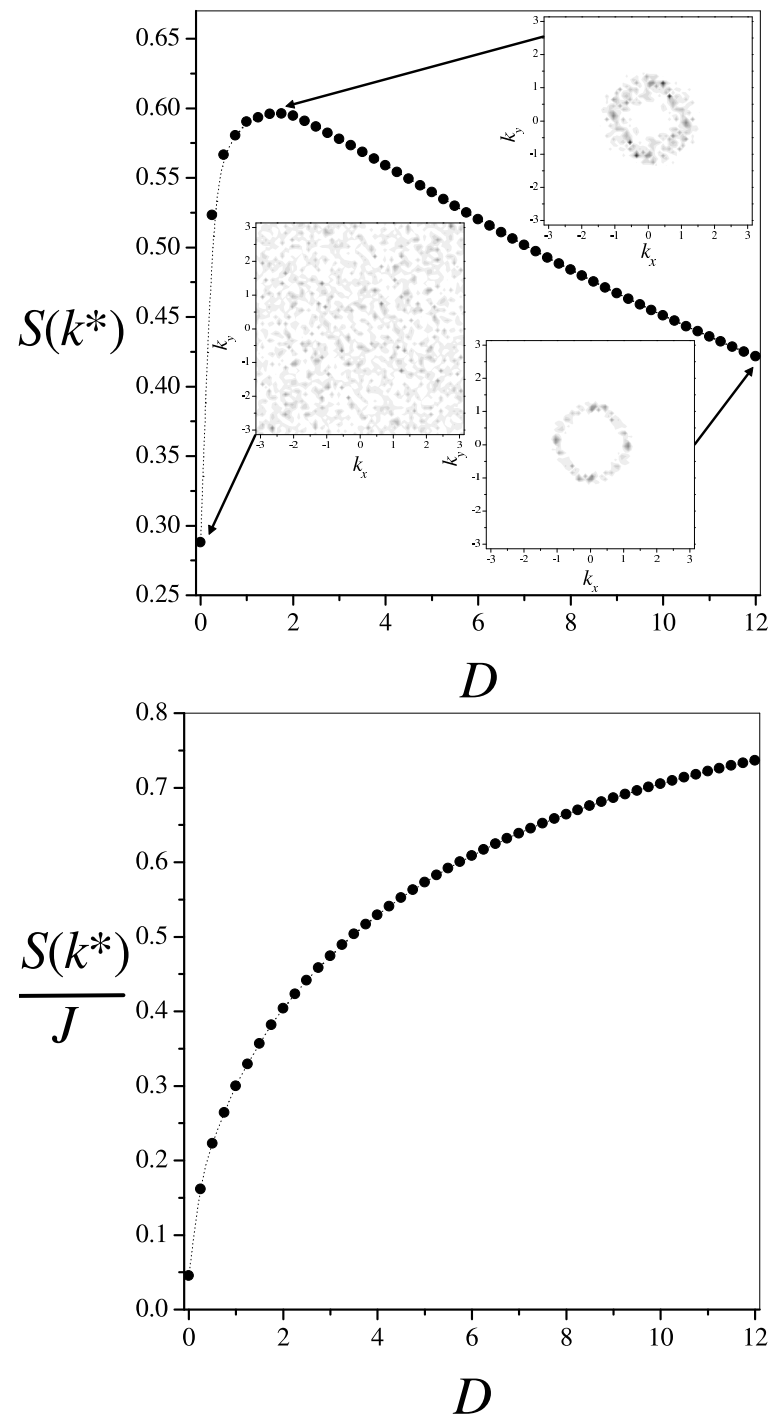

FIG. 7: First panel: re-entrant behavior of the total power spectrum as a function of the coupling for $m=1$ and $\Delta=2.5$. The inset panels show $\chi(\mathbf{k})$ for $D=0, D=2$, and $D=12$ by means of density plots: the same gray scale is used in all cases. Although $S\left(k^{*}\right)$ shows non-monotonic behavior, the pattern coherence increases as the coupling grows, as seen in the monotonic behavior of the ratio $S\left(k^{*}\right) / J$ in the second panel.

solved analytically within the mean field model. We show that pattern formation can be achieved through continuous (second order) and discontinuous (first order) transitions, and that the pattern formation phenomenon is re-entrant with the coupling. Thus, increasing the coupling eventually destroys the order. All these predictions have been checked by means of numerical simulations, and we find full qualitative (albeit not quantitative) agreement with the theory.

Beyond the capabilities of the modified mean field approach, we have explored not only the occurrence of patterns but also their coherence. The numerical simulations show that even while patterns become stronger (as reflected by a larger value of the total power spectrum at the particular wavevector magnitude characteristic of the pattern), they may become less coherent (as reflected by a larger contribution of neighboring wavevectors). The converse is also possible: patterns that become more coherent even as they weaken.

We end by stressing that the methodology and formalism developed herein can be generalized straightforwardly to other kinds of disorder and/or couplings. 


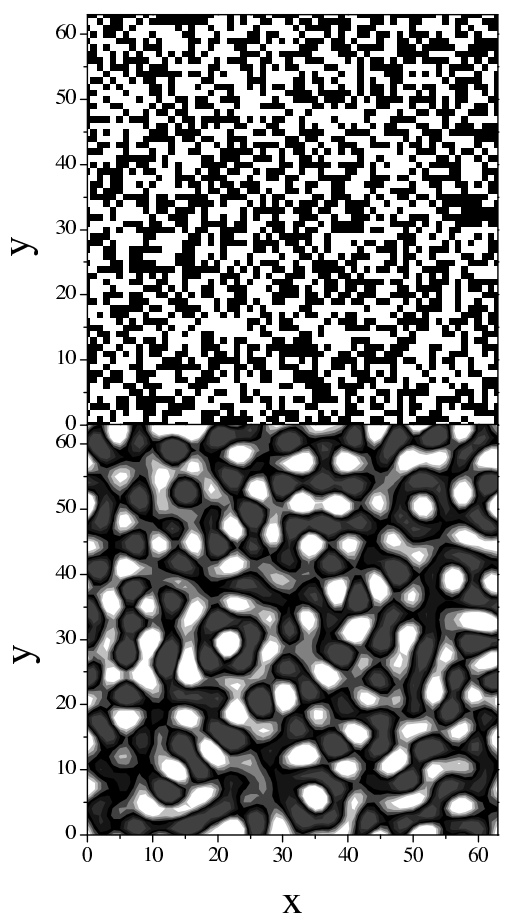

FIG. 8: Density plot showing a stationary pattern purely induced by disorder for $m=1, \Delta=2.5$, and $D=12$ (lower panel). The upper panel shows the underlying configuration of quenched disorder for this particular simulation.

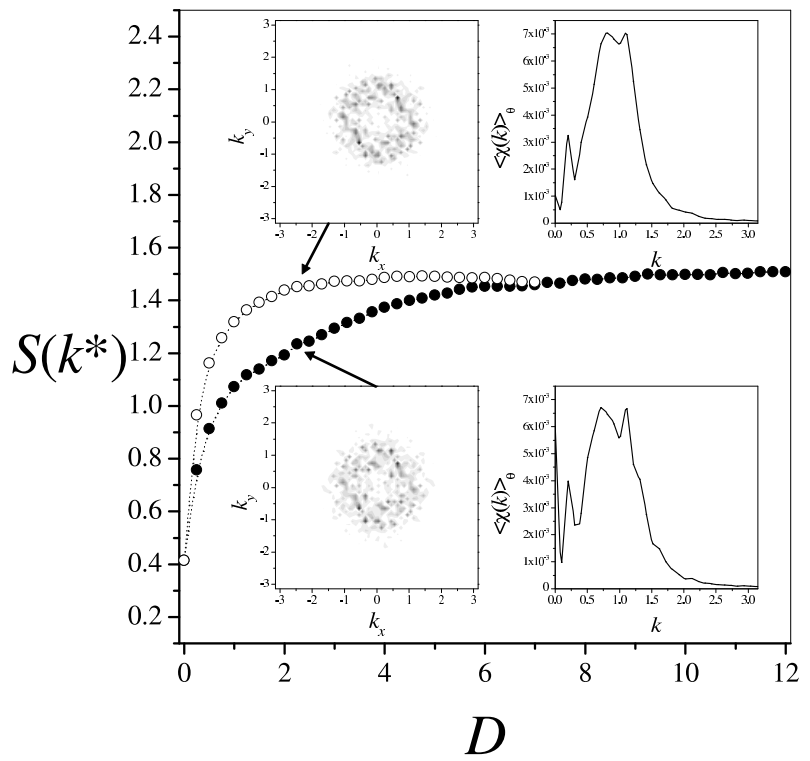

FIG. 9: Total power spectrum as a function of the coupling for $m=2$ and $\Delta=3$. The hysteresis is reflected in the difference between the black and white circle phase space trajectories, obtained when increasing and decreasing $D$ respectively. The insets show density plots of $\chi(\mathbf{k})$ and their angular averages.

\section{Acknowledgments}

We thank J. M. R. Parrondo for fruitful discussions. This work was supported by the Engineering Research Program of the Office of Basic Energy Sciences at the U. S. Department of Energy under Grant No. DE-FG03-86ER13606. Support was also provided by MECD-Spain Grant No. EX2001-02880680, and by MCYT-Spain Grant No. BFM20010291 . 


\section{APPENDIX A: GENERALIZATION OF MODIFIED MEAN FIELD THEORY}

We begin by illustrating some detailed dependences associated with the ansatz field (11) and the action of $\mathcal{L}$ on it. For example, for an $\boldsymbol{r}^{\prime}$ that is $m$ lattice sites away from $\boldsymbol{r}=\left(x_{1}, x_{2}, \ldots, x_{d}\right)$ in the direction $j$ the ansatz reads

$$
\phi\left(x_{1}, x_{2}, \ldots, x_{j}+m \Delta x, \ldots, x_{d}\right)=\sum_{\left\{\boldsymbol{k}^{*}\right\}} \mathcal{A}\left(k^{*}\right) \cos \left(m \Delta x k_{j}\right)+B .
$$

For an $\boldsymbol{r}^{\prime}$ that is in the immediate positive diagonal location away from $\boldsymbol{r}$ we have

$$
\phi\left(x_{1}+\Delta x, x_{2}+\Delta x, \ldots, x_{j}+\Delta x, \ldots, x_{d}+\Delta x\right)=\sum_{\left\{\boldsymbol{k}^{*}\right\}} \mathcal{A}\left(k^{*}\right) \cos \left[\Delta x\left(k_{1}+k_{2}+\cdots+k_{d}\right)\right]+B .
$$

Next, to apply the discrete version (6) of $\mathcal{L}$ we must elucidate the effect of the operators $\left[\sum_{i=1}^{d} \sinh ^{2}\left(\frac{\Delta x}{2} \frac{\partial}{\partial x_{i}}\right)\right]^{n}$ on the field $\phi_{\boldsymbol{r}}$ for $n=1,2$. With $n=1$, we use the relation $2 \sinh ^{2}(y / 2)=[\cosh (y)-1]$ and note that

$$
\begin{aligned}
\sum_{i=1}^{d} \cosh \left(\Delta x \frac{\partial}{\partial x_{i}}\right) \phi_{\boldsymbol{r}}= & \frac{1}{2}[ \\
& \left(x_{1}+\Delta x, x_{2}, \ldots, x_{j}, \ldots, x_{d}\right) \\
& +\phi\left(x_{1}-\Delta x, x_{2}, \ldots, x_{j}, \ldots, x_{d}\right) \\
\ldots & +\phi\left(x_{1}, x_{2}, \ldots, x_{j}+\Delta x, \ldots, x_{d}\right) \\
& +\phi\left(x_{1}, x_{2}, \ldots, x_{j}-\Delta x, \ldots, x_{d}\right) \\
\ldots & +\phi\left(x_{1}, x_{2}, \ldots, x_{j}, \ldots, x_{d}+\Delta x\right) \\
& \left.+\phi\left(x_{1}, x_{2}, \ldots, x_{j}, \ldots, x_{d}-\Delta x\right)\right] .
\end{aligned}
$$

By using Eq. A1 in this last equation we obtain

$$
\sum_{i=1}^{d} \cosh \left(\Delta x \frac{\partial}{\partial x_{i}}\right) \phi_{\boldsymbol{r}}=\sum_{\left\{\boldsymbol{k}^{*}\right\}} \mathcal{A}\left(k^{*}\right) \sum_{i=1}^{d} \cos \left(k_{i} \Delta x\right)+B .
$$

As for $n=2$, we note that $4 \sinh ^{2}(y / 2) \sinh ^{2}(z / 2)=[\cosh (y)-1][\cosh (z)-1]$ and, in turn, $\cosh (y) \cosh (z)=$ $\frac{1}{2}[\cosh (y+z)+\cosh (y-z)]$. The latter combination leads to contributions that involve both forward and backward translations in different spatial directions. This is easily visualized by noting explicitly that

$$
\begin{aligned}
{\left[\sum_{i=1}^{d} \cosh \left(\Delta x \frac{\partial}{\partial x_{i}}\right)\right]^{2}=} & \frac{1}{2}\left[\sum_{i, j=1}^{d} \cosh \left(\Delta x\left(\frac{\partial}{\partial x_{i}}+\frac{\partial}{\partial x_{j}}\right)\right)\right. \\
& \left.+\cosh \left(\Delta x\left(\frac{\partial}{\partial x_{i}}-\frac{\partial}{\partial x_{j}}\right)\right)\right] .
\end{aligned}
$$

Notice that for the $d$ cases where with $i=j$, the second term on the right hand side leaves the field at the original site $\boldsymbol{r}$. The field at the original site is not represented by the ansatz assumption, and therefore we must subtract the $d$ "spurious" terms produced by the ansatz state and add $d$ times the field $\phi_{\boldsymbol{r}}$. This procedure leads to,

$$
\left[\sum_{i=1}^{d} \cosh \left(\Delta x \frac{\partial}{\partial x_{i}}\right)\right]^{2} \phi_{\boldsymbol{r}}=\frac{d}{2} \phi_{\boldsymbol{r}}+\sum_{\left\{\boldsymbol{k}^{*}\right\}} \mathcal{A}\left(k^{*}\right)\left[\left(\sum_{i=1}^{d} \cos \left(k_{i} \Delta x\right)\right)^{2}-\frac{d}{2}\right]-\frac{d}{2} B .
$$

Note that we have taken advantage of the directional insensitivity of $k^{*}$.

Use of Eqs. (A4) and (A6) in Eq. (6) then leads to the following approximation for the term containing the SwiftHohenberg coupling operator:

$$
\mathcal{L} \phi_{\boldsymbol{r}}=D_{1}\left(\sum_{\left\{\boldsymbol{k}^{*}\right\}} \mathcal{A}\left(k^{*}\right)-\phi_{\boldsymbol{r}}\right)+B\left(D_{1}-D k_{0}^{4}\right)
$$


where $D_{1}$ is given in Eq. (13).

Finally, since the summand in Eq. (A7) is independent of the direction of the $\boldsymbol{k}^{*}$, the sums simply give the number of terms $\mathfrak{n}\left(k^{*}\right)$ in the sum, as given in Eq. (10) (or the appropriate integral form), times the summand. Thus we finally arrive at the mean field approximation

$$
\mathcal{L} \phi_{\boldsymbol{r}}=D_{1}\left[\mathfrak{n}\left(k^{*}\right) \mathcal{A}\left(k^{*}\right)-\phi_{\boldsymbol{r}}\right]+B\left(D_{1}-D k_{0}^{4}\right) .
$$

\section{APPENDIX B: NORMALIZATION OF THE FOURIER TRANSFORM AND ORDER PARAMETER}

In this appendix we provide details on the relations between different relevant parameters used in the characterization of pattern formation.

The Fourier transform of a field $\phi_{\mathbf{r}}$ and its inverse read respectively

$$
\begin{aligned}
\widetilde{\phi}_{\mathbf{k}} & =C \sum_{\mathbf{r}} \phi_{\mathbf{r}} e^{-i \mathbf{k} \cdot \mathbf{r}}, \\
\phi_{\mathbf{r}} & =\widetilde{C} \sum_{\mathbf{k}} \widetilde{\phi}_{\mathbf{k}} e^{i \mathbf{k} \cdot \mathbf{r}},
\end{aligned}
$$

where $C$ and $\widetilde{C}$ are normalization constants. Since

$$
\begin{aligned}
& \frac{1}{N^{d}} \sum_{\mathbf{k}} e^{i \mathbf{k} \cdot\left(\mathbf{r}-\mathbf{r}^{\prime}\right)}=\delta_{\mathbf{r}, \mathbf{r}^{\prime}}, \\
& \frac{1}{N^{d}} \sum_{\mathbf{r}} e^{i \mathbf{r} \cdot\left(\mathbf{k}-\mathbf{k}^{\prime}\right)}=\delta_{\mathbf{k}, \mathbf{k}^{\prime}},
\end{aligned}
$$

the functional relation between $C$ and $\widetilde{C}$ can be obtained by substituting Eq. (B1) into Eq. (B2). One readily obtains

$$
\widetilde{C}=\frac{1}{C N^{d}}
$$

For simplicity we choose $C=1 / N^{d}$, and therefore $\widetilde{C}=1$.

Two parameters commonly used to characterize spatial patterns are the total power spectrum, $S(k)$, and the flux of convective heat, $J$,

$$
\begin{aligned}
S(k) & =\sum_{\{k\}} \widetilde{\phi}_{\mathbf{k}} \tilde{\phi}_{-\mathbf{k}}, \\
J & =\frac{1}{N^{d}} \sum_{\mathbf{r}} \phi_{\mathbf{r}}^{2},
\end{aligned}
$$

where the sum in (B6) runs over all modes of magnitude $k$. It is easy to check that the functional relation between these two quantities is simply

$$
J=\sum_{k} S(k),
$$

where now the sum runs over the magnitude of the modes.

For a pure spatial pattern of wave vector of magnitude $k^{*}$ where all spatial directions contribute in the same way we expect $\phi_{\mathbf{r}}$ to be

$$
\phi_{\mathbf{r}}=\sum_{\left\{k^{*}\right\}} \mathcal{A}(k) \cos (\mathbf{k} \cdot \mathbf{r}) .
$$

Therefore, the Fourier transform of such a field is

$$
\widetilde{\phi}_{\mathbf{k}}=\frac{1}{2} \mathcal{A}\left(k^{*}\right) \sum_{\left\{k^{*}\right\}}\left(\delta_{\mathbf{k}, \mathbf{k}^{*}}+\delta_{-\mathbf{k}, \mathbf{k}^{*}}\right) .
$$


Consequently, the total power spectrum and the flux of this field are respectively,

$$
\begin{aligned}
S(k) & =\frac{1}{4} \sum_{\{k\}} \mathcal{A}^{2}\left(k^{*}\right) \sum_{\left\{k^{*}\right\}}\left(\delta_{\mathbf{k}, \mathbf{k}^{*}}+\delta_{-\mathbf{k}, \mathbf{k}^{*}}\right)^{2}=\mathfrak{n}\left(k^{*}\right) \mathcal{A}^{2}\left(k^{*}\right) \delta_{k, k^{*}} . \\
J & =\mathfrak{n}\left(k^{*}\right) \mathcal{A}^{2}\left(k^{*}\right) .
\end{aligned}
$$

[1] P. Meakin, Fractals, Scaling and Growth far from Equilibrium (Cambridge University Press, Cambridge, 1998).

[2] L. Solymar and D. Walsh, Lectures on the Electrical Properties of Materials (Claredon Press, Oxford, 1984).

[3] S.-K. Ma, Modern Theory of Critical Phenomena (Benjamin-Cummings, Reading, 1976).

[4] P. W. Anderson, Phys. Rev. E 109, 1492 (1958).

[5] M. C. Cross and P.C. Hohenberg, Rev. Mod. Phys. 65, 851 (1993).

[6] I. R. Epstein and J. A. Pojman, An Introduction to Nonlinear Chemical Dynamics (Oxford, New York, 1998); C. O. Weiss, Phys. Rep. 219, 311 (1992).

[7] J. Buceta, K. Lindenberg, and J. M. R. Parrondo, Phys. Rev. Lett. 88, 024103 (2002); Phys. Rev. E 66, 036216 (2002); ibid 069902(E) (2002); J. Buceta and K. Lindenberg, Phys. Rev. E 66, 046202 (2002).

[8] J. Swift and P. C. Hohenberg, Phys. Rev. A 15, 319 (1977).

[9] J. García-Ojalvo and J. M. Sancho, Noise in Spatially Extended Systems (Springer, New York, 1999).

[10] J. M. R. Parrondo, C. Van den Broeck, J. Buceta, and F.J. de la Rubia, Physica A 224, 153 (1996).

[11] A. A. Zaikin and L. Schimansky-Geier, Phys. Rev. E 58, 4355 (1998).

[12] J. Buceta, M. Ibañes, J. M. Sancho, and K. Lindenberg, Phys. Rev. E 67, 021113 (2003).

[13] C. Van den Broeck, J. M. R. Parrondo, and R. Toral, Phys. Rev. Lett. 73, 3395 (1994); C. Van den Broeck, J. M. R. Parrondo, R. Toral, and R. Kawai, Phys. Rev. E 55, 4084 (1997).

[14] S. Mangioni, R. Deza, H. S. Wio, and R. Toral, Phys. Rev. Lett. 79, 2389 (1997).

[15] S. Kim, S. H. Park, and C. S. Ryu, Phys. Rev. E 58, 7994 (1998).

[16] J. Buceta, J. M. R. Parrondo, and F. J. de la Rubia, Phys. Rev. E 63, 031103 (2001). 